\title{
Monitoring the Fabrication of Tapered Optical Fibres
}

\author{
K. Mullaney, R. Correia ${ }^{\#}$, S. E. Staines, S. W. James* and R. P. Tatam, \\ Engineering Photonics, Cranfield University, \\ Bedford, MK43 0AL, UK.
}

\begin{abstract}
A variety of optical methods to enhance the process of making optical fibre tapers are explored. A thermal camera was used to both refine the alignment of the optical components and optimize the laser power profile during the tapering process. The fibre transmission was measured to verify that the tapers had the requisite optical characteristics while the strain experienced by the fibre while tapering was assessed using an optical fibre Bragg grating. Using these techniques, adiabatic tapers were fabricated with a $2 \%$ insertion loss.
\end{abstract}

Keywords: Optical, taper, sensor, $\mathrm{CO}_{2}$ laser, fibre Bragg grating, laser material processing, adiabatic.

\section{INTRODUCTION}

Tapered optical fibres can be used as sensors for a broad range of bio-medical and chemical applications ${ }^{1}$. A tapered fibre is produced by heating a short length of optical fibre while pulling it under tension. This results in a length of fibre which has a reduced diameter waist in the heated region. In the region of the taper waist, the evanescent field of the propagating mode expands into the media around the fibre, so permitting the modal light to interact with media located within the sensing zone around the taper waist. The transmitted light is sensitive to refractive index changes within the proximity of the taper waist and this property forms the basis of tapers being used as interferometric sensors ${ }^{2,3}$. Tapered optical fibres used in bio-chemical sensing applications usually have diameters in the range 5 to $20 \mu \mathrm{m}^{1}$. These taper waist diameters are readily achievable using a $\mathrm{CO}_{2}$ laser as the heat source. The optical properties of tapers are highly sensitive to the taper's physical geometry and so the ability to make reproducible and low loss tapers is important. To understand and optimize the tapering process for bio-medical sensing applications, the use of in-situ process monitoring techniques was viewed as beneficial.

The objective of this paper is to investigate methods that can be used together to refine the process features of importance in the manufacture of tapers ${ }^{4}$. The methods investigated are: imaging of the heated taper using a thermal camera, measuring the visible and near infra-red (NIR) light transmitted via the taper using a spectrophotometer and the use of a fibre Bragg grating (FBG) as a strain sensing device. The information obtained from this work is also relevant to taper fabrication techniques using alternative fibre heating methods.

\section{FIBRE TAPER FABRICATION SYSTEM}

The optical fibre taper fabrication system employed is shown in figure 1, together with the co-ordinate definition. The output from a Synrad 48-2 $\mathrm{CO}_{2}$ laser, with a maximum output power of $25 \mathrm{~W}$, was used to heat the fibre. ZnSe optical elements were used for power tapping and injecting the HeNe alignment laser into the path of the $\mathrm{CO}_{2}$ laser beam. The galvanometer mounted mirror (Cambridge Technology 6210) was driven with a $100 \mathrm{~Hz}$ triangular waveform to provide uniform illumination along the section of fibre to be tapered. A ZnSe plano-convex cylindrical lens with a $100 \mathrm{~mm}$ focal length, in conjunction with a predetermined increasing laser power, was used to achieve the required energy flux at the fibre, so allowing the softening temperature to be maintained as the fibre diameter decreased ${ }^{5}$. Two programmable rotation stages (Physik Instrumente) supported and located the fibre to be heated and stretched.

\footnotetext{
${ }^{*}$ s.w.james@cranfield.ac.uk; phone +441234754623; http:// www.cranfield.ac.uk/centres/engineering-photonics-centre \# current address Department of Electrical and Electronic Engineering, University of Nottingham, NG7 2RD, UK
}

\footnotetext{
This paper was published in 25th International Conference on Optical Fiber Sensors and is made available with permission of SPIE. One print or electronic copy may be made for personal use only. Systematic electronic or print reproduction and distribution, duplication of any material in this paper for a fee or for commercial purposes, or modification of the content of the paper are prohibited.
} 
The operation of the laser, galvanometer and rotation stages were controlled using a program written in LabVIEW. A NIR camera was used to aid the alignment of the heated fibre within the focused laser spot and to observe both the thermal intensity distribution along the fibre and the displacement of the fibre during the pulling process.

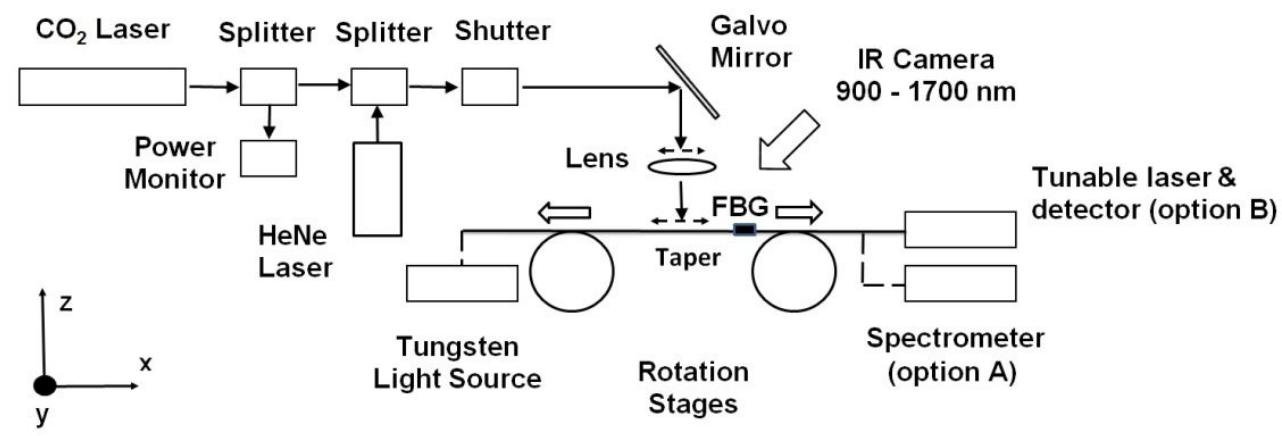

Figure 1. Schematic of experimental taper system using a $\mathrm{CO}_{2}$ laser.

The transmission spectrum of the fibre was monitored using a CCD spectrophotometer and tungsten light source over the wavelength range $500-1150 \mathrm{~nm}$. This allowed the process to be terminated when the desired taper transmission spectrum was achieved (option A in figure 1). Finally, the strain experienced by the fibre was monitored during the heat and stretch process using an FBG (option B in figure 1).

\section{EXPERIMENTAL RESULTS AND DISCUSSION}

To optimize and to ensure repeatability of the process, three monitoring techniques were used.

\subsection{NIR Camera}

The NIR camera was used for two purposes. The first was to determine the laser power profile required to maintain the fibre at the melting point over the duration of the tapering process. As the fibre diameter decreases during the taper fabrication, so the laser power has to be increased to maintain the required temperature. To determine this laser power, the heated fibre was imaged by the NIR (Vosskuhler NIR-300P) camera, and the laser power that caused the fibre axial length to expand rapidly was noted. This occurs near the fibre softening temperature $(\sim 1183 \mathrm{~K}){ }^{6}$ and causes the fibre to displace across the camera field of view in the z-direction.

The second purpose was to ensure that the scanning focal spot was aligned with the longitudinal axis of the fibre, thus maintaining a uniform temperature. This was practically achieved by adjusting the fibre position in the y-axis (figure 1) to ensure that the intensity recorded by the camera along the scanned length of the fibre was uniform and at the intensity peak position. The spectral response of the camera covers the range $900-1700 \mathrm{~nm}$ and is sensitive to the black-body thermal emission spectra from the heated fibre. The camera spectral response and calculated Planck radiated power curves ${ }^{7}$ for a range of temperatures near the fibre melting point of $1183 \mathrm{~K}$ are shown in figure 2 . The camera integrates the overlap of the two response curves so the intensity of the fibre image is related to the temperature of the fibre. Use of the NIR camera to align the system was critical in ensuring that symmetric tapers could be fabricated. A typical symmetric adiabatic taper produced by this system is illustrated in figure 3 .

\subsection{Optical fibre Bragg gratings}

$\mathrm{CO}_{2}$ lasers are prone to mode-hopping when the operating conditions (power, temperature) change ${ }^{8,9}$. This would be expected to cause rapid changes in the fibre temperature, which might cause the fibre to cool below the softening temperature and influence detrimentally the fabricated taper. An in-line FBG, interrogated using a tunable laser (option $\mathrm{B}$ in figure 1), was used to monitor the strain applied to the fibre during the taper fabrication process. The strain measured by the FBG during taper fabrication is shown in figure 4, together with the measured and programmed 


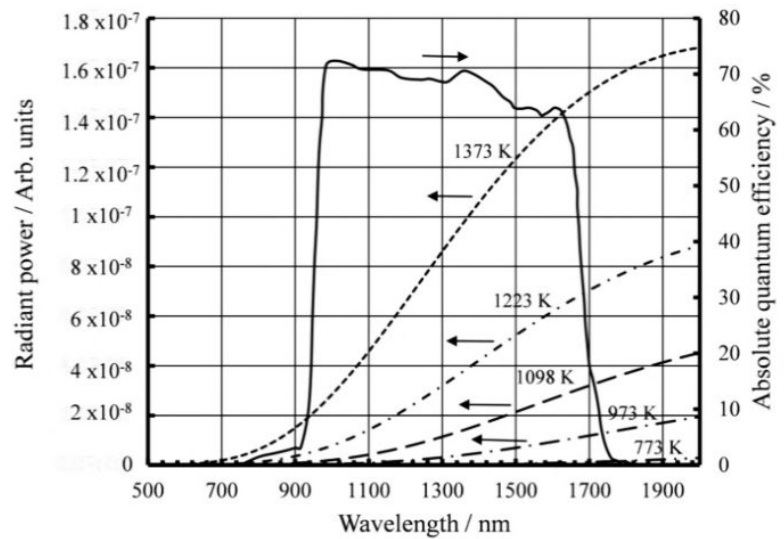

Figure 2: The NIR camera spectral response (solid line) and Planckian black body radiation curves (dashed lines) for a range of temperatures near the fibre melting point of $1183 \mathrm{~K}$

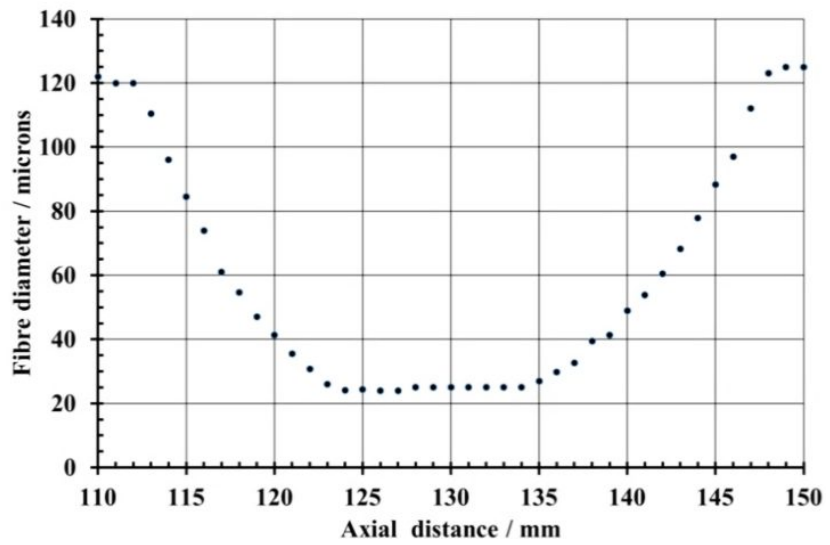

Figure 3: The profile of an adiabatic taper. The taper waist diameter was $25 \mu \mathrm{m}$, the fibre used was SM750.

variation in laser power. The fluctuations in laser power are a result of mode hopping. At $102 \mathrm{~s}$, the fibre was stretched, resulting in an abrupt increase in the fibre strain. As the laser power increases, the fibre starts to soften, resulting in a subsequent decrease in strain. As the fibre was then heated and pulled, no significant change in the strain was observed until $180 \mathrm{~s}$ elapsed. After this time, the strain experienced by the fibre is modulated in sympathy with the power fluctuations, suggesting that as the laser power falls it is not sufficient to maintain the reduced-diameter fibre at the softening temperature, and so the fibre solidifies and the strain increases. In this application, FBGs can be used to; precisely time the onset of fibre pulling, optimizing the $\mathrm{CO}_{2}$ laser power profile and identifying when fibre breakage is imminent.

\subsection{Monitoring the transmission spectrum}

A CCD spectrophotometer and a tungsten light source were used to measure the transmission spectrum of the fibre over the wavelength range 500-1150 nm (option A in figure 1). Figure 5 shows the evolution of the channeled transmission spectrum [1] of a non-adiabatic taper with a diameter of order $13 \mu \mathrm{m}$, showing the development of the characteristic channeled spectrum that results from the interference between the modes excited in the tapered region.

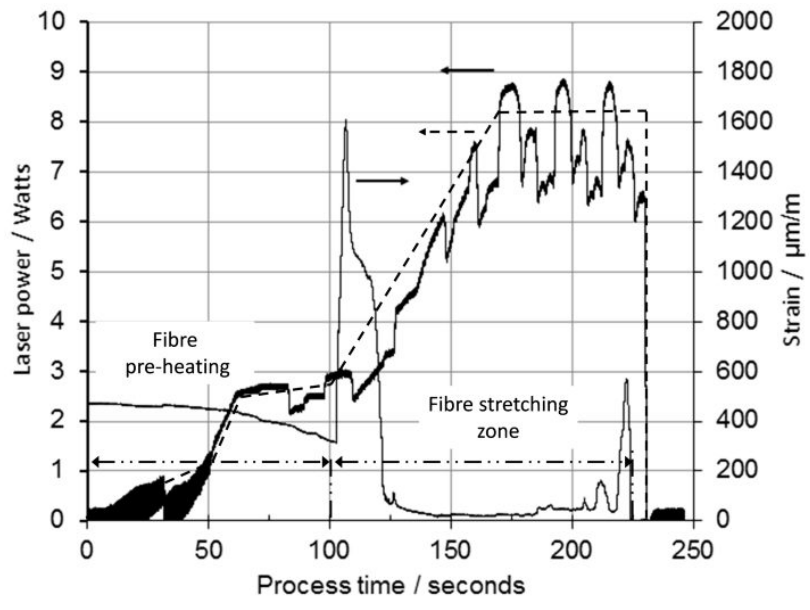

Figure 4: The strain measured by an FBG and laser power during the fabrication of a taper. The dashed line is the programmed laser power and the dashed/dotted lines defines the fibre heating and stretching zones.

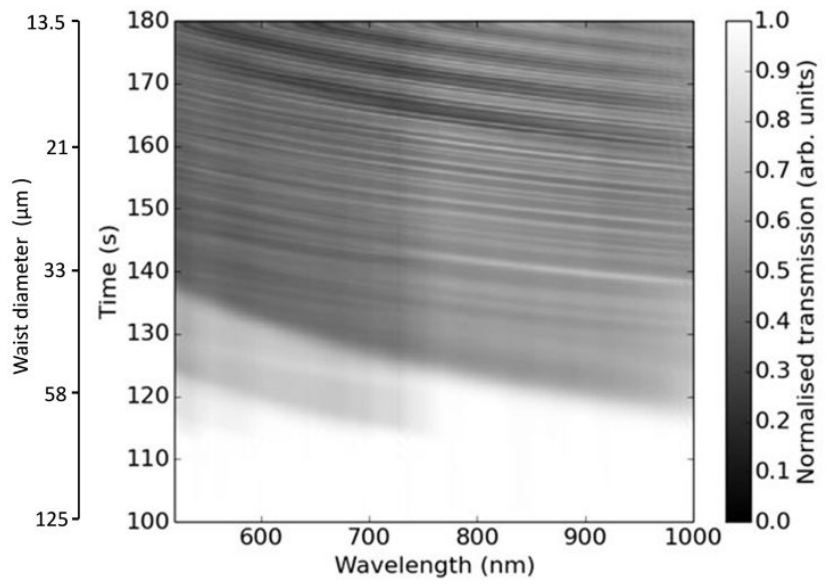

Figure 5: Evolution of the channelled spectrum during the fabrication of a non-adiabatic $13 \mu \mathrm{m}$ taper. 


\subsection{Typical taper profile}

The information provided by these measurement techniques has allowed the optimization of the laser power cycle, system alignment and has confirmed the importance of stability of the laser power when fabricating tapered fibers with small waists. In addition, it facilitates the reproducible fabrication of tapers with a desired spectral characteristic. A typical adiabatic taper profile produced by this system under optimized alignment and power conditions is shown in figure 3. The fibre diameter was measured using an Olympus BX51 microscope at $1 \mathrm{~mm}$ intervals along the tapered fibre. The taper is $36 \mathrm{~mm}$ long with an average waist diameter of $25.0 \pm 0.5 \mu \mathrm{m}$. The transition zones were $\sim 12 \mathrm{~mm}$ long with a taper angle of $2.8 \pm 0.1$ mrads. The transmission loss of this taper was $2 \%$. Ten of these tapers were made with a typical waist variation of $\pm 0.5 \mu \mathrm{m}$ and similar transmission losses, indicating that the process was repeatable.

\section{SUMMARY \& CONCLUSIONS}

During the course of this work, a variety of different tapers were fabricated. These tapers had waist diameters down to 13 $\mu \mathrm{m}$ and waist lengths of $\sim 10 \mathrm{~mm}$ using single-mode SMF-28 fibre. A range of optical techniques to monitor the process of fabricating optical fibre tapers has also been investigated. A camera operating in the near infra-red has been used both to aid fibre alignment and to allow the laser power profile for the process to be easily determined.

Monitoring the transmission spectrum of the fibre during the tapering process allows the operator to ensure that the taper will have the required spectral properties in the wavelength region of interest. The use of an IR camera, together with monitoring the transmission of the fibre, are effective process tools that allows the taper process to be optimized for specific sensing applications.

FBGs have been used to measure strain during the stretching process and can be used to precisely time the onset of fibre pulling, optimizing the $\mathrm{CO}_{2}$ laser power profile and identifying when fibre breakage is imminent. In this context, FBGs can be used for developing and optimizing the tapering process but not for routine taper production, as using FBGs increases fibre handling time and could reduce the mechanical integrity of the tapered fibre.

\section{ACKNOWLEDGEMENTS}

The work was supported by funding from the Engineering and Physical Sciences Research Council (EPSRC) UK (EPL010437, EP-H02252X). Data underlying this study can be accessed through the Cranfield University repository at http://dx.doi.org/10.17862/cranfield.rd.3969222.

\section{REFERENCES}

1. Jarzebinska, R., Cheung, C., James S. W., and Tatam, R. P., "Response of the transmission spectrum of tapered optical fibres to the deposition of a nanostructured coating," Meas. Sci. Technol., 20, 034001 (2009).

2. Lacroix, S., Gonthier, F., Black R. and Bures, J., "Tapered-fibre interferometric wavelength response- the achromatic fringe," Opt. Lett,. 13, 395-397 (1988).

3. Gonthier, F., Lapierre, J., Veilleux, C., Lacroix, S., and Bures, J., "Investigation of power oscillations along tapered monomode fibres," Appl. Opt., 26, 444-449 (1987).

4. Mullaney, K., Correia, R., Staines, S., James S.W. and Tatam, R. P., "Monitoring techniques for the manufacture of tapered optical fibres," Appl. Opt., 54, 8531-8536 (2015).

5. Sumetsky, M., Dulashko Y. and Hale, A., "Fabrication and study of bent and coiled free silica nanowires: Selfcoupling microloop optical interferometer," Opt. Express, 12, 3521-353 (2004).

6. Bayle F., and Meunier, J., "Efficient fabrication of a fused -fibre biconical taper structures by a scanned $\mathrm{CO}_{2}$ laser beam technique," Appl. Opt. 44, 6402-6411 (2005).

7. Rees, W., "Physical Principles of Remote Sensing", $2^{\text {nd }}$ edition, Cambridge University (2001).

8. Li, B., Wang, Y., Peng D. and Li, Q., "Power measure and control system for high power laser in real-time," Proc. SPIE, 6622, 66221 (2007).

9. Zheng, W., Sugawara, H., Mizushima T. and Klimowych, W., "Heating power feedback control for $\mathrm{CO}_{2}$ laser fusion splicers," Proc. SPIE, 8601, 860129 (2013). 
2017-04-23

\section{Monitoring the fabrication of tapered optical fibres}

Mullaney, Kevin

SPIE

K. Mullaney; R. Correia; S.E. Staines; S.W. James; R.P. Tatam. Monitoring the fabrication of tapered optical fibres. 25th International Conference on Optical Fiber Sensors, 24-28 April 2017, Jeju, Republic of Korea. Paper number: 103232D http://dx.doi.org/10.1117/12.2263285

Downloaded from Cranfield Library Services E-Repository 